\title{
Skin Toxicity Ant Anti-EGFR-Therapy: A Mock Trouble for Elderly and Frail Patients with Mcrc
}

\author{
Raffaele Addeo* and Gaetano Cimmino \\ U.O.C. Oncologia, "S. Giovanni di Dio" Hospital, \\ ASLNA2NORD Frattamaggiore, Italy \\ *Corresponding author: Raffaele Addeo, U.O. C. \\ Oncologia, "S. Giovanni di Dio" Hospital, ASLNA2NORD \\ Via Pirozzi 80027 Frattamaggiore, Naples, Italy
}

Received: September 03, 2018; Accepted: September 24, 2018; Published: October 01, 2018

\section{Editorial}

Colorectal Cancer (CRC) is mainly a disease of the elderly with a median age at diagnosis of 71 years. At the time of diagnosis, around $20 \%$ of patients have metastatic disease, and about $50-60 \%$ of patients will finally develop metastatic or advanced disease, with a poor 5-year survival rate of $5 \%$.

Elderly patients represent more than half of all individuals suffering from Colorectal Cancer (CRC) in Western countries and their prevalence is continuously increasing [1]. The treatment of these patients has been controversial over the last years. Today, we know that the efficacy and toxicity of chemotherapy in elderly patients with good PS is not influenced by age. For this reason, senescence was defined as the passage of biological time, and ageing as the passage of chronological time. Usually, age of 70 years is considered a reference point and is commonly used in clinical trials in oncology.

There is no clear and universal definition of 'frail' elderly patient, but generally, the following criteria are accepted: age $>85$ years, dependence for one or more Activities of Daily Living (ADL), >3 significant associated conditions and presence of one or more typical geriatric syndromes such as incontinence in the absence of stress, dementia, depression [2]. This setting of people has a mean life expectancy of around 2 years.

Given the toxicity of chemotherapies, there is no doubt that the 'frail' elderly population with $\mathrm{mCRC}$ should not receive standard chemotherapy treatments due to potential side effects, including the possibility of toxicity death, exceed the potential benefits. In the clinical practice, several factors such as PS, co morbidity, age or perhaps social networks influence the choice to carry out chemotherapy. Co morbidities may substantially influence the diagnostic work-up, treatment intensity and modalities, thereby affecting prognosis and survival. Increased co morbidity is seen in patients with advanced disease. These patients have been historically excluded or underrepresented in most clinical trials for this reason we do not have sufficient evidence on the appropriate management of elderly patients with metastatic CRC. The development of new therapeutic agents like bevacizumab (anti-VRGF) and cetuximab or panitumumab (anti-Epidermal Growth Factor Receptor (EGFR)) which target specific molecular events in tumour cells provides new opportunities to improve the treatment of this type of cancer.
Furthermore, these new-targeted biological therapies have fewer systemic toxic effects and potentially could be suitable in frail patients.

Skin toxicity is a class-specific side effect and it is related to the inhibition of EGFR in the skin, which is critical for the normal evolution and physiology of the epidermis. Acne form skin eruptions are the most frequent side effect of EGFR inhibitors and it typically manifested as a papulopustular rash in the majority (45-100\%) of patients receiving EGFR inhibitors. Other cutaneous reactions consisted of painful fissures; Xerox is, in palms and soles, alterations in hair growth, and microsites. However, an early prophylaxis and therapeutic interventions for each type of toxicity were strongly suggested and it guarantees a reduction in the severity of the adverse event. In a single-arm, multicentre, phase II trial including only frail patients, Sastre evidenced that panitumumab achieved a 6-month PFS rate was $36.4 \%$ with an objective response rate of $9.1 \%$. The study confirm the efficacy and safety of anti EGFR therapy and it may be a therapeutic option for high-risk frail elderly patients with WT mCRC considered not candidates for chemotherapy [3]. Oncologists must carefully discern between PS and co morbidity when assessing patients for therapy. For patients with good PS, restricting cetuximab or panitumumab use in the setting of significant co morbidity does not appear justified [4].

The reduced performance status did not prevent oncologists from treating pts with anti EGFR agent-containing chemotherapy schedules. Elderly pts with reduced performance status and co morbidities can be treated effectively and safely with cetuximab. In a large Non-Interventional Studies (NISs) help to evaluate therapies in daily practice, Jehn and colleagues showed a similar efficacy of Cetuximab was combined with irinotecan in the two groups of patients selected for their age. Among the 657 recruited patients, the PFS did not differ between pts 18-65 years old (6.5 months) in comparison with pts 465 years (7.0 months). $64 \%$ of the pts developed skin rash, without any difference between age groups [5]. Age cannot represent an obstacle to the use of antiEGFR therapy on the other hand, we do not use it as a predictor of increased skin toxicity. The addition of anti-EGFR to chemotherapy in first- or second-line RAS WT mCRC does not negatively impact on overall QoL, despite the occurrence of skin toxicity. In a recent analysis developed on three randomized clinical trials, the authors underline that skin toxicity of a worst grade of $>3$ appeared to have similar impact on QoL outcomes as skin toxicity of grade $<3$. While skin toxicity had no impact on overall QoL [6]. On the other hand, the skin toxicity, especially rash, paronychia, and pruritus, represent a clinically relevant adverse event that requires proactive management. Our current knowledge the importance of establishing a pre-emptive, comprehensive skin toxicity program in patients treated with anti EGFR-therapy [7-9]. It is clear that the reduction of the severity of skin toxicities, it determines an improvements in QoL, lack of interference on antitumor effect. This prophylactic action causes 
a decreased need for dose modification a d justify this therapeutic rationale. The use of a complete geriatric evaluation could be useful in order to differentiate fragile patients and those at high risk of toxicity from the rest of the elderly population, in order to help the doctor choose the best therapeutic strategy. We cannot preconceive exclude these patients from treatment with anti-EGFR.

A thorough and objective analysis of the PS, of the co morbidities rather than the age, as well as the life expectancy of the patient, may drive the physician to choice this therapeutic option. Our current knowledge suggest and continue to promote the EGFR inhibitors also in the elderly and frail patients with mCRC because of their efficacy and safety as well as positive impact on quality of life.

\section{References}

1. Siegel R, Naishadham D, Jemal A. Cancer statistics. CA Cancer J Clin. 2012 62: 10-29.

2. Pal SK, Katheria V, Hurria A. Evaluating the older patient with cancer: understanding frailty and the geriatric assessment. CA Cancer J Clin. 2010; 60: 120-132.

3. Sastre J, Massuti B, Pulido G. First-line single-agent panitumumab in frail elderly patients with wildtype KRAS metastaticcolorectal cancer and poor prognostic factors: A phase II study of the Spanish CooperativeGroup for the Treatment of Digestive Tumours. Eur J Cancer. 2015: 1371-1380.
4. Asmis TR, Powell E, Karapetis CS. Comorbidity, age and overall survival in cetuximab-treated patients with Advanced Colorectal Cancer (ACRC)- results from NCIC CYG CO. 17: a phase III trial of cetuximab versus best supportive care. Ann Oncol. 2011; 22: 118-126.

5. Jehn CF, Böning L, Kröning H, Possinger K, Lüftner D. Cetuximab-based therapy in elderly comorbid patients with metastatic colorectal cancer. $\mathrm{Br} \mathrm{J}$ Cancer. 2012; 106: 274-278

6. Koukakis R, Gatta F, Hechmati G, Siena S Skin toxicity and quality of life during treatment with panitumumab for RAS wild-type metastatic colorectal carcinoma: results from three randomised clinical trials. Qual Life Res. 2016; 25: 2645-2656.

7. Kobayashi Y, Komatsu Y, Yuki S, Fukushima H. Randomized controlled trial on the skin toxicity of psanitumumab in Japanese patients with metastatic colorectal cancer HGCSG1001 study; J-STEPP. Future Oncol. 2015; 11: 617-627.

8. Lacouture ME, Mitchell EP, Piperdi B, Pillai MV, Shearer H, lannotti N, et al. Skin Toxicity Evaluation Protocol with Panitumumab (STEPP), a phase II, open-label, randomized trial evaluating the impact of a pre-Emptive Skin treatment regimen on skin toxicities and quality of life in patients with metastatic colorectal cancer. J Clin Oncol. 2010; 28: 1351-1357.

9. Thaler J, Karthaus M, Mineur L. Skin toxicity and quality of life in patients with metastatic colorectal cancer during first-line panitumumab plus FOLFIRI treatment in a single-arm phase II study. BMC cancer 2012; 12: 248.
Ann Hematol Oncol - Volume 5 Issue 6 - 2018

ISSN : 2375-7965 | www.austinpublishing group.com

Addeo et al. (C) All rights are reserved
Citation: Addeo R and Cimmino G. Skin Toxicity Ant Anti-EGFR-Therapy: A Mock Trouble for Elderly and Frail Patients with Mcrc. Ann Hematol Oncol. 2018; 5(6): 1213. 